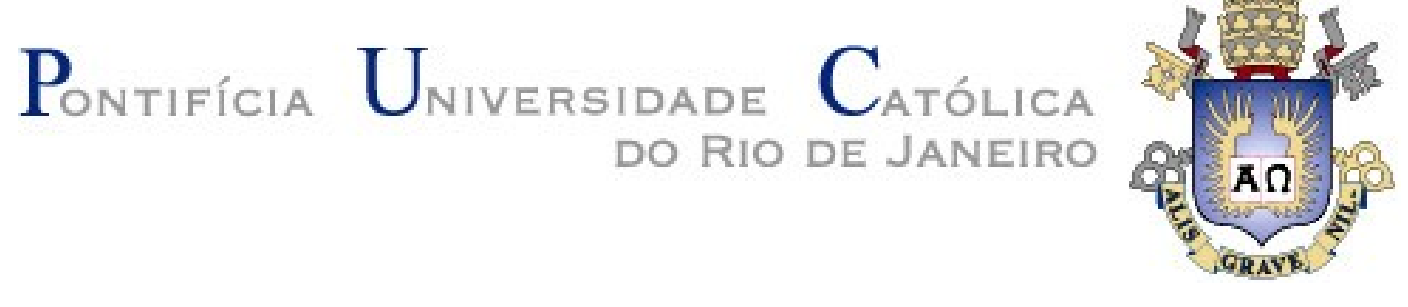

Andrea Isabel Rojas Eraso

\title{
Análise de Confiabilidade de Longarinas de Pontes Ferroviárias de Concreto Armado
}

\begin{abstract}
Dissertação de Mestrado
Dissertação apresentada como requisito parcial para obtenção do grau de Mestre pelo Programa de PósGraduação em Engenharia Civil da PUC-Rio.

Orientadora: Prof. ${ }^{a}$ Marta de Souza Lima Velasco Co-orientadora: Prof. ${ }^{a}$ Andréia A. Diniz de Almeida
\end{abstract}

Rio de Janeiro 


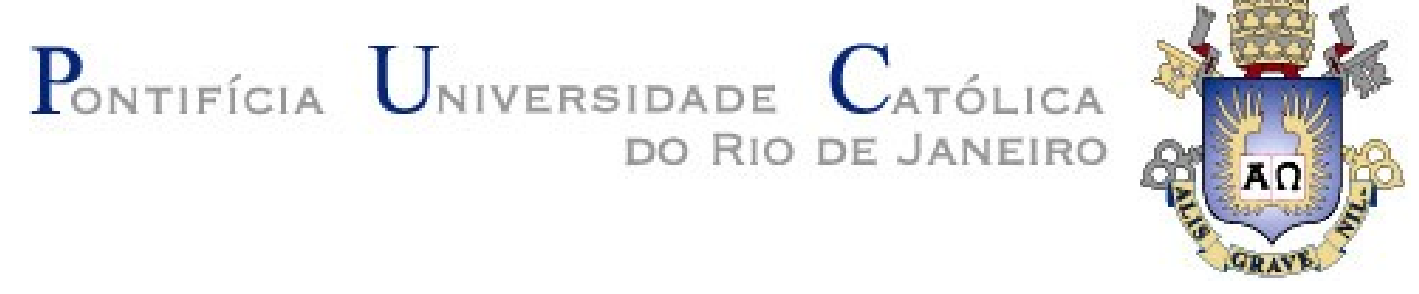

Andrea Isabel Rojas Eraso

\title{
Análise de Confiabilidade de Longarinas de Pontes \\ Ferroviárias de Concreto Armado
}

Dissertação apresentada como requisito parcial para obtenção do grau de Mestre pelo Programa de Pós-Graduação em Engenharia Civil da PUC-Rio. Aprovada pela Comissão Examinadora abaixo assinada.

\author{
Prof. ${ }^{a}$ Marta de Souza Lima Velasco \\ Orientador \\ Departamento de Engenharia Civil - PUC-Rio \\ Prof. ${ }^{a}$ Andréia A. Diniz de Almeida \\ Co-orientadora \\ Universidade Federal Fluminense \\ Prof. ${ }^{a}$ Claudia Maria de Oliveira Campos \\ Universidade Federal Fluminense \\ Prof. Rodrigo Bird Burgos \\ Departamento de Engenharia Civil - PUC-Rio \\ Prof. José Eugenio Leal \\ Coordenador Setorial do Centro \\ Técnico Científico - PUC-Rio
}

Rio de Janeiro, 9 de setembro de 2011 
Todos os direitos reservados. É proibida a reprodução total ou parcial do trabalho sem autorização da universidade, do autor e do orientador.

\section{Andrea Isabel Rojas Eraso}

Graduou-se em Engenharia Civil na Universidade de Nariño, Pasto - Colombia, em 2008. Atua na área de Confiabilidade estrutural com ènfase em concreto armado.

Ficha Catalográfica

Rojas Eraso, Andrea Isabel

Análise de confiabilidade de longarinas de pontes ferroviárias de concreto armado / Andrea Isabel Rojas Eraso; orientadora: Marta de Souza Lima Velasco; coorientadora: Andréia A. Diniz de Almeida - 2011.

128 f. : il. (color.) ; $30 \mathrm{~cm}$

Dissertação (mestrado)-Pontifícia Universidade Católica do Rio de Janeiro, Departamento de Engenharia Civil, 2011.

Inclui bibliografia

1. Engenharia Civil - Teses. 2. Concreto armado. 3. Confiabilidade de estruturas. 4. Estado limite último. 5. Confiabilidade de estruturas. I. Velasco, Marta de Souza Lima. II. Almeida, Andreia A Diniz. III. Pontificia Universidade Católica do Rio de Janeiro. Departamento de Engenharia Civil. IV. Título. 
"Não há sabedoria alguma, nem discernimento algum, nem plano algum que possa opor-se ao Senhor. Prepara-se o cavalo para o dia da batalha, mas o Senhor é que dá a vitória”. Prov. 21:30-31 


\section{Agradecimentos}

A Deus pelo seu amor e sua fidelidade, nada teria sentido sem sua maravilhosa presença.

Aos meus pais e a minha família pelo seu amor, seu apoio, suas orações, por ter sempre as palavras oportunas no momento certo.

A minha orientadora Marta de Souza Lima Velasco, pelos conhecimentos transmitidos e por toda sua colaboração.

A Andréia A. Diniz minha co-orientadora, pelos conhecimentos transmitidos, disponibilidade, paciência, incentivo e principalmente pela amizade desenvolvida ao longo destes anos.

Aos professores do departamento, especialmente ao professor Rodrigo Bird Burgos pelos ensinamentos e colaboração neste trabalho.

A todos os amigos e colegas que me acompanharam nesta caminhada, pelos bons momentos compartilhados.

A Lorena Chamorro pela sua amizade e seu apoio, pelas longas noites de trabalhos e por cada momento compartilhado juntas.

A todas as pessoas que direta ou indiretamente, contribuíram para a realização deste trabalho, cada aporte foi fundamental.

A CAPES e a PUC-Rio pelo apoio financeiro. 


\section{Resumo}

Rojas Eraso, Andrea Isabel; Velasco, Marta de Souza Lima; Almeida, Andreia A. Diniz. Análise de Confiabilidade de Longarinas de Pontes Ferroviárias de Concreto Armado, Rio de Janeiro, 2011. 128 p. Dissertação de Mestrado Departamento de Engenharia Civil, Pontifícia Universidade Católica do Rio de Janeiro.

Em todo projeto de estruturas de engenharia existem incertezas associadas às propriedades dos materiais, às propriedades geométricas e aos carregamentos. Essas incertezas geralmente são consideradas através de fatores de segurança. A análise de confiabilidade aplicada ao projeto de estruturas é uma ferramenta que permite avaliar a probabilidade de falha da estrutura para um determinado modo de comportamento e a sensibilidade deste projeto em relação às variáveis consideradas. Neste trabalho são aplicadas estratégias de avaliação da confiabilidade das vigas principais de uma ponte existente de concreto armado, as quais são verificadas no estado limite último na flexão simples e no estado último de serviço na formação de fissuras, segundo as recomendações da NBR6118:2003. Foram desenvolvidas rotinas com o auxílio do programa Matlab para avaliar a probabilidade de falha da ponte segundo o método de simulação de Monte Carlo e o método FORM (First Order Reliability Method). Também é realizada uma análise de sensibilidade para analisar a influência de cada variável na confiabilidade da ponte.

\section{Palavras-chave}

Pontes Ferroviárias; confiabilidade; concreto armado; estruturas existentes. 


\section{Abstract}

Rojas Eraso, Andrea Isabel; Velasco, Marta de Souza Lima (Advisor); Almeida, Andreia A. Diniz (Co-Advisor). Reliability Analysis for Stringers of Concrete Railway Bridges. Rio de Janeiro, 2011. 128p. MSc. Dissertation - Departamento de Engenharia Civil, Pontifícia Universidade Católica do Rio de Janeiro.

In the project of engineering structures, some design variables are usually taken as deterministic, although there are uncertainties associated with loads, material and geometrical properties. The use of safety factors is the most common strategy to deal with these uncertainties. The reliability analysis of structures is a tool to assess the probability of structural failure in a certain behavior and the sensitivity of this failure in relation to each variable considered. This work is concerned with the reliability analysis of the main beams of an existing bridge made of reinforced concrete, which is checked at the ultimate limit state in simple bending and the ultimate state of service in the formation of cracks as recommended by NBR6118: 2003. Matlab routines are developed in order to assess the probability of failure of the bridge using two methods: Monte Carlo simulation and FORM (First Order Reliability Method). Additionally, a sensitivity analysis is performed in order to analyze the influence of each variable in the reliability of the bridge.

\section{Keywords}

Railway bridges; reliability; reinforced concrete; existing structures. 


\section{Sumário}

1 Introdução 21

1.1. Considerações Iniciais 21

1.2. Objetivo 22

1.3. Organização do Trabalho 23

2 Revisão Bibliográfica $\quad 25$

2.1. Estruturas Existentes 25

2.2. Monitoração e Gerenciamento de Pontes 29

2.3. Fadiga 33

2.4. Pontes de Concreto Armado 35

2.5. Códigos de Calibração 36

2.6. Modelos de Carga 38

2.7. O FORM Como Método de Avaliação da Probabilidade de Falha 39

2.8. Programas Computacionais 40

3 Sistemática de Avaliação de Pontes e Viadutos 41

3.1. Panorama Geral do que é Feito 41

3.2. Normas Técnicas 44

4 Confiabilidade Estrutural 45

4.1. Introdução $\quad 45$

4.2. Estados Limites $\quad 48$

4.3. Função de Estado Limite 49

4.4. Probabilidade de Falha 49

4.5. Métodos de Cálculo da Probabilidade de Falha 51

4.5.1. Método de Primeira Ordem FORM (First Order Reliability Method) 52

4.5.2. Análise de Sensibilidade 56

4.5.3. Método de Simulação de Monte Carlo 56

4.6. Índice de Confiabilidade de Referência 58

5 Formulação do Problema 61 
5.1. Introdução 61

5.2. Verificação de Segurança no Estado Limite Último 61

5.3. Variáveis Aleatórias $\quad 62$

5.4. Função de Estado Limite $\quad 64$

5.5. Momento Resistente 64

5.6. Momento Solicitante 68

5.7. Verificação de Segurança no Estado Limite de Serviço 71

5.7.1. Estado Limite de Formação de Fissuras 71

5.7.2. Estado Limite de Abertura de Fissuras $\quad 74$

5.8. Rotinas Implementadas para Análise de Confiabilidade Associadas ao

Estado Limite de Ruptura $\quad 79$

6 Estudo de Caso 83

6.1. Descrição Geral da Ponte 83

6.2. Análise de Confiabilidade da Ponte 84

6.2.1. Análise com Seis Variáveis Aleatórias 86

6.2.2. Análise com Quatro Variáveis Aleatórias 88

6.2.3. Análise com Três Variáveis Aleatórias 93

6.2.4. Influência do Coeficiente de Variação (COV) da Carga Móvel (Q) na Probabilidade de Falha $\quad 94$

6.2.5. Influência da Variação da Carga Móvel na Probabilidade de Falha 97

6.2.6. Análise com Quatro Variáveis Aleatórias sem Considerar Coeficientes de Segurança 99

6.2.7. Influência da Variação COV da Carga Móvel (Q) na Probabilidade de $\begin{array}{ll}\text { Falha } & 101\end{array}$

6.2.8. Influência da Variação da Carga Móvel na Probabilidade de Falha 103

6.3. Análise no Estado Limite de Serviço na Formação de Fissuras 105

7 Conclusões e Sugestões 107

7.1. Sugestões 108

8 Referências Bibliográficas 109

$\begin{array}{ll}\text { Anexo A Teoria de Probabilidade } & 119\end{array}$

A.1. Introdução 119

$\begin{array}{ll}\text { A.2. Variável Aleatória } & 119\end{array}$

A.3. Função Cumulativa de Distribuição (CDF) e Função Densidade de 
$\begin{array}{ll}\text { Probabilidade (PDF) } & 119\end{array}$

A.4. Principais Parâmetros de uma Variável Aleatória Contínua 121

A.5. Distribuições de Probabilidade 122

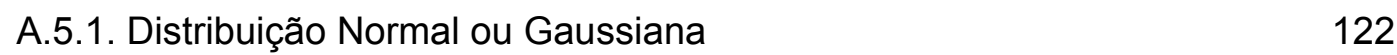

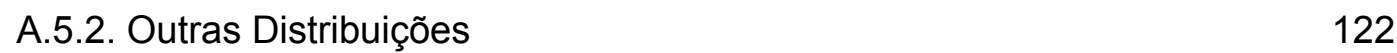

$\begin{array}{ll}\text { A.5.3. Distribuições Normais Equivalentes } & 124\end{array}$

$\begin{array}{ll}\text { A.5.4. Coeficientes de Correlação Equivalentes } & 124\end{array}$

A.6. Coeficientes Parciais de Segurança 125

$\begin{array}{ll}\text { A.7. Valores Característicos das Variáveis } & 126\end{array}$ 


\section{Lista de figuras}

Figura 2.1. Pesquisas necessárias e áreas de estudo envolvidas (adaptado de Catbas et al 2008)

Figura 2.2. Esquema básico para análise de confiabilidade usando

SHM (adaptado de Catbas et al 2008 ). 32

Figura 4.1 Representação da integral de convolução (fonte: Melchers 2002) 50

Figura 4.2. Definição do domínio de falha (fonte: Melchers 2002).

Figura 4.3. Transformação do espaço original para o espaço reduzido normal padrão (fonte: Choi e Youn 2001).

Figura 4.4. Aproximação do Método FORM para superfícies côncavas e convexas (fonte: Lopez 2007).

Figura 4.5. Representação gráfica da busca do ponto de projeto para um problema com duas variáveis (fonte: Choi e Youn 2001).

Figura 5.1. Domínios de estado limite último de uma seção transversal (fonte: NBR 6118:2003)

Figura 5.2. Seção Tipo da ponte 66

$\begin{array}{ll}\text { Figura 5.3. Esquema geral para uma viga } T & 67\end{array}$

Figura 5.4. Locomotiva tipo DASH9 (fonte: Relatório Técnico,

Veloso et al 2007). 69

Figura 5.5. Vagão tipo GDT (fonte: Relatório Técnico, Veloso et al 2007). 69

Figura 5.6. Esquema geral dos estádios de deformação. 72

Figura 5.7. Concreto de envolvimento da armadura (fonte NBR6118:2003) 78

Figura 5.8. Fluxograma esquemático das opções de análise

implementadas no programa de confiabilidade de estruturas.

82

Figura 6.1. Vista geral da ponte sobre o Rio Vermelho

(fonte: Relatório Técnico, Veloso et al 2007).

Figura 6.2. Sistema estrutural da ponte (fonte: Relatório Técnico, Veloso et al 2007).

Figura 6.3. Seção $\pi$ da ponte sobre o Rio Vermelho (a) largura da longarina $35 \mathrm{~cm}$. (b) Largura da longarina $70 \mathrm{~cm}$. (fonte: Relatório Técnico, Veloso et al 2007).

Figura 6.4. Comparação do índice de confiabilidade obtido pelo FORM 
para 6 e 4 variáveis aleatórias.

Figura 6.5. Comparação da probabilidade de falha obtida pelo FORM

para 6 e 4 variáveis aleatórias.

Figura 6.6. Comparação da probabilidade de falha para as análises

feitas com seis, quatro e três variáveis aleatórias.

Figura 6.7. Variação do índice de confiabilidade em função do COV da carga móvel $Q$.

Figura 6.8. Variação do fator de importância em função do COV de Q.

Figura 6.9. Comparação do índice de confiabilidade em função

da variação de $Q$

Figura 6.10. Comparação da probabilidade de falha em função

da variação de $Q$

98

Figura 6.11. Fator de importância em função da variação da carga móvel Q. 99

Figura 6.12. Comparação da probabilidade de falha obtida

com e sem coeficientes de segurança

100

Figura 6.13. Variação do índice de confiabilidade em função do COV da carga móvel $Q$, sem coeficientes de segurança.

Figura 6.14. Variação do fator de importância em função do COV de $Q$, sem coeficientes de segurança.

Figura 6.15. Comparação do índice de confiabilidade em função da variação de $Q$ sem coeficientes de segurança

Figura 6.16 Probabilidade de falha em função da variação de $Q$ sem coeficientes de segurança

Figura A.1 (a) Função Densidade de Probabilidade (PDF) e

(b) Função Cumulativa de Distribuição (CDF).

Figura A.2. Valor característico típico para a variável Resistência S

(fonte: James 2003)

Figura A.3. Valor característico típico para a variável solicitação $S$

(fonte: James 2003) 


\section{Lista de tabelas}

Tabela 2.1. Níveis de avaliação da segurança, (Wisniewski, 2007)

Tabela 4.1. Valores do índice de confiabilidade de referência $\beta_{\mathrm{T}} \mathrm{e}$

Probabilidade de falha $P_{f}$ associadas, relacionadas ao estado limite último.

Tabela 4.2. Valores do índice de confiabilidade de referência $\beta_{T} e$

probabilidade de falha $P_{f}$ associados ao estado limite de serviço

60

Tabela 5.1. Modelos probabilísticos das variáveis aleatórias

Tabela 5.2. Abertura máxima das fissuras $\left(w_{k}\right)$, para combinação freqüente, em função das classes de agressividade ambiental (NBR6118:2003).

Tabela 5.3. Classes de agressividade ambiental

Tabela 6.1. Seções consideradas na análise

Tabela 6.2. Dados probabilísticos das variáveis aleatórias

Tabela 6.3. Dados de área e momento de inércia para as seções estudadas

85

Tabela 6.4. Armaduras de tração e compressão para cada seção

Tabela 6.5. Probabilidade de falha segundo os métodos: simulação de Monte Carlo e FORM para seis variáveis aleatórias sem considerar armadura de pele.

Tabela 6.6 Comparação das probabilidades de falha calculadas segundo a simulação de Monte Carlo e o FORM sem armadura de pele para 6 variáveis aleatórias.

Tabela 6.7. Probabilidade de falha segundo os métodos: simulação de Monte Carlo e FORM para seis variáveis aleatórias com armadura de pele

Tabela 6.8. Comparação das probabilidades de falha calculadas segundo a simulação de Monte Carlo e o FORM com armadura de pele para 6 variáveis aleatórias.

Tabela 6.9. Probabilidade de falha para os métodos: simulação de Monte Carlo e FORM para quatro variáveis aleatórias sem armadura de pele. 89 Tabela 6.10. Comparação das probabilidades de falha calculadas segundo a simulação de Monte Carlo e o FORM sem armadura de pele para 4 variáveis aleatórias.

Tabela 6.11. Resultado da probabilidade de falha para os métodos: simulação de Monte Carlo e FORM para quatro variáveis aleatórias com armadura de pele 
Tabela 6.12. Comparação das probabilidades de falha calculadas segundo a simulação de Monte Carlo e o FORM sem armadura de pele para 4 variáveis aleatórias

Tabela 6.13. Comparação entre as análises feitas com o FORM para seis e quatro variáveis aleatórias

Tabela 6.14. Valores característicos e valores médios das variáveis aleatórias

Tabela 6.15. Resultados do método FORM para quatro variáveis aleatórias

Tabela 6.16. Comparação entre as análises feitas com o FORM

para quatro variáveis aleatórias.

Tabela 6.17. Resultado do método FORM para três variáveis aleatórias

Tabela 6.18 Comparação entre as análises com três e seis variáveis aleatórias.

Tabela 6.19. Análise de sensibilidade da probabilidade de falha em função do COV da carga móvel $Q$.

Tabela 6.20. Valores característicos e valores médios da carga móvel $Q$

Tabela 6.21. Resultado do FORM para quatro variáveis aleatórias

com carga móvel Q aumentada $25 \% 50 \%$ e $100 \%$

Tabela 6.22. Analises para quatro variáveis aleatórias pelo método FORM, considerando a armadura de pele. 100

Tabela 6.23. Resultados obtidos com e sem coeficientes de segurança 100 Tabela 6.24. Análises para quatro variáveis aleatórias pelo método FORM, desconsiderando a armadura de pele.

Tabela 6.25. Comparação entre os dados obtidos com e sem coeficientes de segurança, sem armadura de pele.

Tabela 6.26. Resultados da análise de sensibilidade da probabilidade de falha em função do COV da carga $Q$, sem considerar coeficientes de segurança

Tabela 6.27. Resultado do método FORM variando a carga móvel, sem considerar coeficientes de segurança

Tabela 6.28. Resultados (via FORM), para o estado limite de formação de fissuras 


\section{Lista de Símbolos}

\section{Romanos}

\begin{tabular}{|c|c|}
\hline a & Parâmetro da distribuição Uniforme \\
\hline$A_{c r i}$ & Área da região de envolvimento \\
\hline As & Área da seção transversal da armadura longitudinal de tração \\
\hline A's & Área da seção da armadura longitudinal de compressão \\
\hline b & Parâmetro da distribuição Uniforme \\
\hline$b_{f}$ & Largura da mesa da viga \\
\hline$b_{w}$ & Largura da alma da viga \\
\hline C & Valor da confiabilidade da estrutura \\
\hline d & Altura útil \\
\hline d' & Altura útil \\
\hline$E_{c s}$ & Módulo de elasticidade secante do concreto \\
\hline$E_{s}$ & Módulo de elasticidade do aço \\
\hline$E(X)$ & Valor médio ou a média de uma variável aleatória X \\
\hline $\mathrm{F}$ & Domínio de falha \\
\hline $\mathrm{F}$ & $\begin{array}{l}\text { Valor que depende somente de } \rho_{\mathrm{ij}} \text { e dos coeficientes de variação das } \\
\text { variáveis aleatórias não normais }\end{array}$ \\
\hline$f_{c d}$ & Resistência de cálculo à compressão do concreto \\
\hline$f_{c k}$ & Resistência à compressão do concreto \\
\hline$f_{c t}$ & Resistência à tração direta do concreto \\
\hline$f_{c t, m}$ & Resistência media do concreto a tração \\
\hline $\mathrm{F}_{\mathrm{d}, \mathrm{ser}}$ & Valor de cálculo das ações para combinações de serviço \\
\hline $\mathrm{F}_{\text {gik }}$ & Valor característico das ações permanentes \\
\hline $\mathrm{F}_{\mathrm{q} 1 \mathrm{k}}$ & Valor característico da ação variável principal direta \\
\hline $\mathrm{F}_{\mathrm{R}}(\mathrm{s})$ & Representa a probabilidade de $\mathrm{R} \leq \mathrm{s}$ \\
\hline$f_{R}$ & Função densidade de probabilidade marginal da resistência \\
\hline$f_{R}(r)$ & Função densidade de probabilidade da resistência \\
\hline$f_{R S}$ & Função densidade de probabilidade conjunta \\
\hline$f_{R S}(r, s)$ & Função conjunta de densidade de probabilidade de R e S \\
\hline$f_{S}$ & Função densidade de probabilidade marginal da solicitação \\
\hline$f_{s}(s)$ & Função densidade de probabilidade da solicitação \\
\hline$f_{s}(s) d s$ & Representa a probabilidade de $S$ assumir um valor entre s e s+ds \\
\hline
\end{tabular}


$F_{X}(X) \quad$ Função cumulativa de distribuição

$\mathrm{f}_{\mathrm{x}}(\mathrm{X}) \quad$ Função densidade de probabilidade

$\mathrm{f}_{\mathrm{yd}} \quad$ Resistência de cálculo à tração do aço

$\mathrm{f}_{\mathrm{yk}} \quad$ Resistência à tração do aço

g(Y) Função de falha escrita em função das variáveis no espaço normal padrão

$g\left(Y^{K}\right) \quad$ Valor da função de falha no espaço reduzido

$\mathbf{G}(\mathbf{X}) \quad$ Função de estado limite no espaço original

$\mathrm{h} \quad$ Altura da viga

$\mathrm{h}_{\mathrm{f}} \quad$ Altura das asas da viga

$\mathrm{I}_{\mathrm{c}} \quad$ Momento de inércia da seção bruta do concreto

$\mathrm{l}_{\mathrm{e}} \quad$ Momento equivalente segundo a formula de Brandson

$\mathrm{I}_{\mathrm{i}} \quad$ Fator de importância

I II Momento de inércia no Estádio II puro

J Jacobiano da transformação de Nataf

k Nível de confiança desejado

k Parâmetro das distribuições Tipo II Máximo e Tipo III Mínimo (Weibull)

$\mathrm{k}_{\mathrm{R}} \quad$ Fator que depende do tipo de distribuição considerada para a resistência e do percentil especificada para o valor característico

$\mathrm{k}_{\mathrm{S}} \quad$ Fator que depende do tipo de distribuição considerada para a solicitação e do percentil especificada para o valor característico

L Matriz triangular inferior obtida a partir da decomposição de Choleski da matriz dos coeficientes de correlações equivalentes das variáveis $x$

$\mathrm{n}_{\mathrm{s}} \quad$ Número de simulações para o método de simulação de Monte Carlo

m Vetor com as médias normais equivalentes das variáveis aleatórias $X$

$\mathrm{M}_{\mathrm{f}} \quad$ Momento de fissuração

$\mathrm{M}_{\mathrm{rd}} \quad$ Momento resistente de cálculo

$M_{s d} \quad$ Momento solicitante de cálculo

$\mathrm{M}_{\mathrm{sp}} \quad$ Momento solicitante por carga permanente

$M_{\text {spadic }} \quad$ Momento solicitante por carga adicional para cálculo do momento por carga permanente

$\mathrm{M}_{\mathrm{sp} 1} \quad$ Momento auxiliar para o cálculo do momento solicitante por carga permanente

$\mathrm{M}_{\mathrm{sq}} \quad$ Momento solicitante por carga móvel 


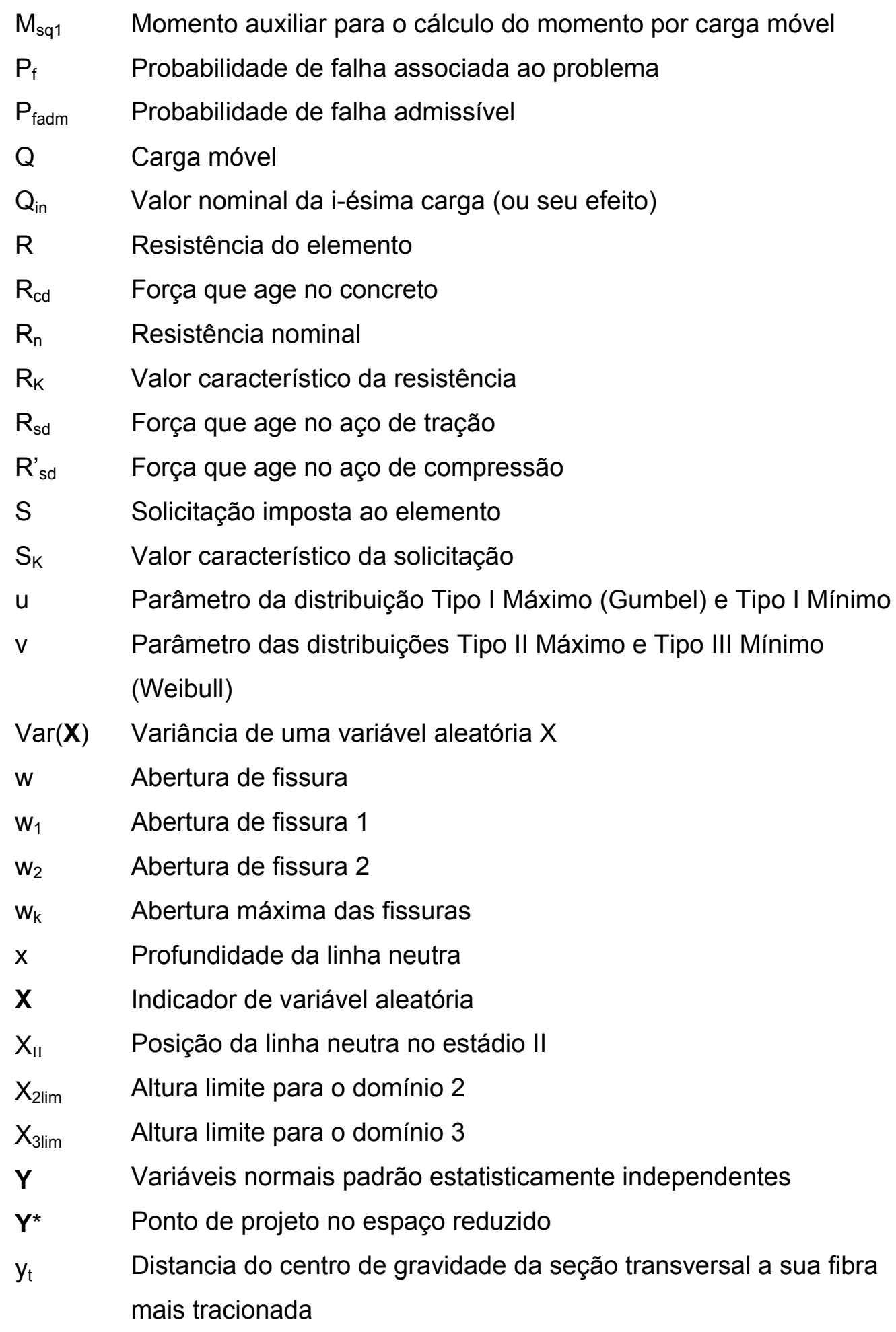

\section{Gregos}

$\alpha \quad$ Parâmetro da distribuição Rayleigh

a Valor que correlaciona aproximadamente a resistência à tração na flexão com a resistência à tração direta

$\alpha_{e} \quad$ Relação entre o módulo de elasticidade do aço e o módulo de 
elasticidade secante do concreto

$\alpha_{i}^{2} \quad$ Co-seno diretor entre o vetor normal à superfície de falha no ponto de projeto e o eixo da variável reduzida

$\beta \quad$ Índice de confiabilidade

$\beta_{\mathrm{adm}} \quad$ Índice de confiabilidade admissível

$\beta_{T} \quad$ Índice de confiabilidade de referência

Г Matriz inversa da matriz L

$\gamma \quad$ Peso específico do concreto

$\gamma_{i} \quad$ Coeficiente de majoração da i-ésima carga (ou seu efeito)

$\gamma_{\mathrm{k}} \quad$ Coeficiente de segurança global ou característico

$\varepsilon_{\mathrm{s}} \quad$ Deformação da armadura

$\varepsilon_{c} \quad$ Deformação da fibra de concreto

$\varepsilon_{\text {yd }}$ Deformação do cálculo do aço correspondente a tensão de escoamento

$\eta \quad$ Coeficiente de conformação superficial

$\lambda \quad$ Parâmetro da distribuição Lognormal

$\lambda_{0} \quad$ Coeficiente de segurança central

$\lambda_{k} \quad$ Coeficiente de majoração da solicitação

$\mu_{R} \quad$ Valor médio da resistência

$\mu_{\mathrm{S}} \quad$ Valor médio da solicitação

$\mu_{\mathrm{X}} \quad$ Valor médio de uma variável aleatória $\mathrm{X}$

$\boldsymbol{\mu}_{x}^{N} \quad$ Média normal equivalente no ponto $x^{*}$

$\xi \quad$ Parâmetro da distribuição Lognormal

$\rho_{i j} \quad$ Coeficiente de correlação entre as variáveis $X_{i}$ e $X_{j}$

$\boldsymbol{\rho}_{i j}^{E} \quad$ Coeficiente de correlação equivalente

$\rho_{\mathrm{r}} \quad$ Taxa de armadura passiva ou ativa aderente em relação à área da região de envolvimento.

$\boldsymbol{\sigma} \quad$ Matriz diagonal contendo os desvios padrões normais equivalentes das variáveis aleatórias $X$

$\sigma \quad$ Tensão obtida pela teoria linear para as cargas máximas esperadas durante a vida útil da estrutura

$\sigma_{\text {adm }} \quad$ Tensão admissível

$\sigma_{\lim } \quad$ Tensão limite

$\boldsymbol{\sigma}_{X}^{N} \quad$ Desvio padrão normal equivalente no ponto $x^{*}$

$\sigma_{\mathrm{s}} \quad$ Tensão de tração no centro de gravidade da armadura considerada 


\begin{tabular}{|c|c|}
\hline$\sigma_{\text {sd }}$ & Tensão de cálculo à compressão no concreto \\
\hline$\sigma_{\text {sd }}^{\prime}$ & Tensão de cálculo á tração no concreto \\
\hline$\sigma_{\mathrm{R}}$ & Parâmetro da distribuição Rayleigh \\
\hline$\sigma_{\mathrm{x}}$ & Desvio padrão de uma variável aleatória $X$ \\
\hline T & Parâmetro das distribuições Tipo I Máximo (Gumbel) e Tipo I Mínimo \\
\hline$\Phi$ & Fator de minoração da resistência \\
\hline$\Phi()$ & Função de distribuição cumulativa da variável normal padrão \\
\hline$\Phi()$ & Função densidade de probabilidade normal padrão \\
\hline$\Phi^{-1}()$ & Inversa da distribuição cumulativa normal padrão \\
\hline$\phi()$ & Representa a função de densidade de probabilidade \\
\hline$\phi$ & Diâmetro da barra utilizada na armadura de tração \\
\hline$\varphi$ & Coeficiente de impacto \\
\hline$\varphi_{\mathrm{k}}$ & Coeficiente de minoração da resistência \\
\hline$\psi_{1}$ & $\begin{array}{l}\text { Fator de redução de combinação freqüente para estado limite de } \\
\text { serviço }\end{array}$ \\
\hline$\psi_{2}$ & $\begin{array}{l}\text { Fator de redução de combinação quase freqüente para estado limite } \\
\text { de serviço }\end{array}$ \\
\hline$\nabla g\left(Y^{K}\right)$ & $\begin{array}{l}\text { Gradiente da função de falha no espaço reduzido, avaliada no ponto } \\
\text { de projeto para iteração } \mathrm{K}, \mathrm{Y}^{\mathrm{K}} \text {. }\end{array}$ \\
\hline$\nabla \mathrm{G}(\mathbf{X})$ & Gradiente da função de falha no espaço original avaliado no ponto $X$ \\
\hline$\nabla g\left(Y^{*}\right)_{i}$ & Componente do gradiente da função de estado no espaço reduzido \\
\hline
\end{tabular}




\section{Lista de Abreviaturas}

AASHTO American Association of State Highway and Transportation Officials ASCE American Society of Civil Engineers

BMS Bridge Management System (Sistema de gerenciamento de pontes)

CEB Comite Euro-International Du Beton

CDF Cumulative Distribution Function (Função Cumulativa de Distribuição)

COV Coeficiente de variação

C.S. Coeficiente de segurança

CVRD Companhia Vale do Rio Doce

EFC Estrada de Ferro Carajás

ELF Estado limite de fadiga

ELS Estado limite de serviço

ELU Estado limite último

FORM First Order Reliability Method (Método de Confiabilidade de Primeira Ordem)

FOSM First Order Second Moment (Método de Confiabilidade de primeira ordem e segundo momento)

HLRF Algoritmo de Hasofer, Lind, Rackwitz e Fiessler

ISO International Organization for Standardization

JCSS Joint Committee on Structural Safety (Comitê de Segurança Estrutural)

LRFD Load Factor Design

LRFD Load and Resistance Factor Design

NBR Norma Brasileira Registrada

NiCAE Núcleo de Instrumentação e Computação Aplicada à Engenharia

PDF Probability Density Function (Função Densidade de Probabilidade)

RSM Response Surface Methodology (Métodos de superfície de resposta)

SHM Structural Health Monitoring (Monitoramento da Saúde Estrutural)

SORM Second Order Realibility Method (Método de Confiabilidade de Segunda Ordem)

VA Variável Aleatória 